\title{
UNIVERSIDAD, SOCIEDAD Y CONOCIMIENTO REFLEXIONES PARA EL DEBATE
}

\author{
Norberto Fernández LAMARRA*
}

Recebido: 16 jun. 2014

Aprovado: 31 jul. 2014

* Universidad Nacional de Tres de Febrero. Buenos Aires, Argentina. E-mail: nflamarra@fibertel.com.ar

Resumen: El artículo presenta algunas reflexiones sobre la relación actual entre universidad, sociedad y políticas del conocimiento en Latinoamérica. Para ello, parte de considerar la situación pasada y presente de la educación superior en la región pero a la vez plantea sus interrogantes mirando hacia el futuro de los niños y jóvenes que hoy transitan las aulas de los sistemas educativos. Se consideran 4 ejes de análisis: "la Universidad que América Latina hubiese tenido", "la Universidad que tenemos", "Universidad y sociedad: su democratización" y "Universidad y conocimiento: la innovación". A través de ellos, y partiendo de un diagnóstico de la situación actual de los sistemas educativos en la región, sus mandatos fundacionales y sus promesas aún no cumplidas, se postula la necesidad de innovar. A modo de ejemplo, se plantea una reflexión a partir de los primeros resultados de una investigación desarrollada por el NIFEDE (UNTREF) sobre la innovación en las universidades públicas en Argentina. El artículo concluye con algunas propuestas para una nueva Universidad en América Latina que se plantean en dos dimensiones. En primer lugar, una serie de desafíos que deben enfrentar las políticas para la educación superior en la región y en segundo lugar los desafíos que deben enfrentar las propias instituciones de nivel superior para que esas políticas logren los resultados que la Sociedad del Conocimiento hoy demanda a la Universidad.

Palabras chave: Universidad. Latinoamérica. Universidad y sociedad. Políticas del conocimiento. Innovación. Desafíos para la región. Estrategias institucionales.

\section{UNIVERSIDADE, SOCIEDADE E CONHECIMENTO: REFLEXÕES PARA O DEBATE}

Resumo: O artigo apresenta algumas reflexões sobre a relação entre a universidade, a sociedade e a política do conhecimento na América Latina. Para fazê-lo, considera a situação passada e presente do ensino superior na região, mas também coloca questões referentes ao futuro das crianças e jovens que hoje frequentam os sistemas de educação. Quatro eixos de análise são considerados: "a Universidade que a América Latina poderia ter tido", "a Universidade que temos", "Universidade e sociedade: sua democratização" e "Universidade e conhecimento: Inovação" Através destes, e com base em um diagnóstico da situação atual dos sistemas de ensino da região, seus mandatos fundadores e as promesas ainda não cumpridas, a necessidade de inovar é postulada. Por exemplo, uma reflexão a partir dos primeiros resultados de uma pesquisa realizada pela NIFEDE (UNTREF) sobre inovação nas universidades públicas na Argentina surge. O artigo conclui com algumas propostas para uma nova universidade na América Latina com base em duas dimensões. Em primeiro lugar, uma série de desafios que enfrentam as políticas para a educação superior na região e em segundo lugar os os desafios que as próprias instituições de nível superior devem enfrentar para que essas políticas alcancem os resultados que a Sociedade do Conhecimento exige da Universidade.

Palavras-chave: Universidade. América Latina. Universidade e sociedade. Políticas do conhecimento. Inovação. Desafíos para a região. Estratégias institucionais.

\section{UNIVERSITY, SOCIETY AND KNOWLEDGE: THOUGHTS FOR DEBATE}

Abstract: The article presents some reflections on the relationship between university, society and knowledge policy in Latin America. To do so, the past and present situation of higher education in the región are considered, along with questions regarding the future of children and youth who are currently attending the education systems. Four axes of analysis are considered: "the Univer- 
sity that Latin America could have had", "the University we do have", "University and society: democratization" and "University and Knowledge: Innovation". Through these, and based on a diagnosis of the current situation of the education systems of the region, its founding mandates and promises yet to be fulfilled, the need to innovate is postulated. For example, a reflection based on the first results of a survey conducted by NIFEDE (UNTREF) on innovation in public universities in Argentina emerges. The paper concludes with some proposals for a new university in Latin America based on two dimensions. Firstly, a number of challenges facing policies for higher education in the region and secondly the challenges that institutions of higher education must face so that these policies achieve the results that the Knowledge Society requires from the University.

Key words: University. Latin America. University and society. Knowledge policy. Innovation. Challenges for the region. Institutional strategies.

\section{INTRODUCCIÓN}

En los últimos 20/30 años se han registrado en la mayor parte de los países de América Latina importantes situaciones -algunas positivas, otras negativas- sobre la relación entre la educación, la universidad, la sociedad y las políticas de conocimiento.

La totalidad de los países de América del Sur -que habían sufrido dictaduras militares- recuperaron la plenitud de su democracia y produjeron cambios significativos en las políticas económicas, sociales y educativas, que se reflejaron -de muy diversa manera- en la Universidad. Varios de ellos -en el marco de procesos democráticos- fueron afectados por gobiernos que pusieron en marcha políticas neoliberales; esos mismos países -y otros- han tenido más recientemente gobiernos que hicieron énfasis en las políticas sociales y educativas de inclusión, con resultados muy diversos.

Ante esta situación -diversa y rica en matices políticos e ideológicos, muchas veces enfrentados- considero imprescindible reflexionar, investigar, debatir sobre la Universidad en América Latina y en cada uno de nuestros países. En algunos de ellos -como, por ejemplo, Brasil, México y más recientemente, Chile- se llevan adelante tanto en el ámbito académico como en el político debates que posibilitan centrar la atención en el futuro de la universidad. En otros -como en el mío, Argentina- esos debates son muy escasos y están restringidos a un pequeño sector de carácter académico y no se amplían a otros ámbitos institucionales y de gobierno, y a la sociedad en su conjunto.

Deseo hacer especial énfasis en el imprescindible debate sobre el futuro de la educación y de la universidad, ya que las políticas educativas y universitarias deben plantearse pensando en ese futuro. Los estudiantes de nuestras escuelas primarias y secundarias (educación básica en algunos países) serán relativamente jóvenes y estarán en pleno ejercicio de sus profesiones dentro de 25/30 años ¿se puede pensar en nuestras sociedades del 2040 o del 2050, 
en cuanto a conocimiento y a desarrollo social y productivo? Difícil... pero imprescindible para diseñar y debatir las políticas sobre nuestros sistemas educativos y universitarios.

Para plantear algunas ideas y propuestas para ese debate imprescindible desde ya siempre limitado porque deberá enriquecerse con el aporte de todosme planteo los siguientes ejes temáticos para desarrollar muy sintéticamente:

- $\quad$ La Universidad que América Latina hubiese tenido

- $\quad$ La Universidad que tenemos

- Universidad y sociedad: su democratización

- Universidad y conocimiento: la innovación

- Algunas propuestas para una nueva universidad en América Latina

\section{LA UNIVERSIDAD QUE AMÉRICA LATINA HUBIESE TENIDO}

En el año 1912 se sanciona en la Argentina una ley de voto secreto y obligatorio para elegir a las autoridades y legisladores nacionales y provinciales. A los cuatro años -en 1916- es elegido Presidente Hipólito Yrigoyen, representando a un importante sector social emergente de la nueva sociedad argentina y desplazando a los sectores oligárquicos que habían monopolizado el poder político en las décadas anteriores.

En las universidades se reflejaba ese predominio de la oligarquía dominante, particularmente en la Universidad Nacional de Córdoba. Por eso, en 1918, los estudiantes de Córdoba se rebelan contra sus autoridades y contra la mayoría de su claustro académico y plantean -a través de un Manifiesto Liminar- sus importantes reivindicaciones en materia política, social y, muy especialmente, universitaria.

Este Movimiento Reformista de los estudiantes cordobeses -que luego se extendió muy rápidamente a todas las universidades argentinas- dio lugar a la probablemente más importante reforma en materia de educación y de universidad en toda América Latina y constituyó una definición importantísima para la Universidad en la región, distinguiéndola del resto del mundo.

La Reforma Universitaria de 1918 planteó una serie de temas centrales, la mayoría de los cuales tienen plena vigencia en la actualidad, a casi un siglo de su elaboración. Muchos de ellos, lamentablemente, son todavía deudas significativas de la universidad latinoamericana.

A modo de síntesis podemos señalar a los siguientes como los temas centrales de la Reforma Universitaria de 1918: 
- La autonomía de la Universidad: éste se ha constituido -desde 1918en el eje central de la universidad en Argentina y en la mayoría de los países latinoamericanos, a diferencia de los sistemas universitarios de América del Norte y de Europa. La autonomía universitaria -establecida en la Constitución Nacional de Argentina en su reforma de 1994- se ha mantenido fuertemente en un sinónimo de democracia en nuestros países. Lamentablemente ha sido violada durante las dictaduras militares sufridas en décadas pasadas por varios países de América del Sur. ${ }^{1}$

- Participación en el gobierno universitario de docentes y estudiantes: éste eje ha sido sustancial para que la autonomía se constituya en un ámbito democrático. En la formulación inicial en 1918 no se preveía la participación de los graduados porque en ese momento los graduados eran parte de la oligarquía a desplazar. Posteriormente, fueron incluidos, al igual que -en algunos estatutos universitarios- los representantes del personal no docente. Las universidades han sido reticentes, en general, en posibilitar la incorporación en su gobierno a representantes de la comunidad en que cada institución se desempeña. Este tema lo retomaremos más adelante

- Cuestionamiento de la universidad como "fábrica" de exámenes y títulos profesionales: este planteo formula un severo cuestionamiento de carácter académico y pedagógico. Ya hace 100 años los estudiantes criticaron a una universidad que sólo brindaba títulos profesionales y que no les daba a los estudiantes una formación académica de carácter científico. Todavía esto no ha sido plenamente superado, lamentablemente, y continúa predominando un modelo de universidad "napoleónico", fuertemente deformado.

- Innovación pedagógica: este eje -como el anterior- muestran la preocupación de los estudiantes de Córdoba, hace un siglo, en plantear la necesidad de que una profunda reforma en lo pedagógico se constituya en un imperativo para pensar una universidad basada en el conocimiento.

1 Para complementar este análisis de la autonomía universitaria en América Latina, ver el artículo de Fernández Lamarra y Perez Centeno (2010). En dicho libro se podrán consultar otros textos sobre el tema de especialistas latinoamericanos como Carlos Tünnermann Berheim, Axel Didniksson, Elia Marun, Victor Rosario Muñoz y otros. Para ampliar sobre las concepciones académico-políticas sobre la autonomía universitaria, ver también Fernández Lamarra y Coppola (2014). 
- Libertad de cátedra y designación de los profesores a través de sistemas de concursos abiertos, que posibiliten el acceso, permanencia y ascenso de los docentes, en oposición a la designación arbitraria y por herencia, propios de los regímenes autocráticos y no democráticos.

- Función social de la universidad y compromiso con el cambio: en la propuesta de la Reforma Universitaria se plantea -desde su inicioun cambio profundo en cuanto a perseguir un modelo tendiente a que las instituciones se comprometieran fuertemente con la sociedad, desarrollando una propuesta de compromiso con el desarrollo social y poniéndose a su servicio. Este principio es lo que ha llevado a plantear a la extensión universitaria como una función principal del sistema universitario. Esto implicó -ya hace 100 años atrás- el desarrollo de sus funciones de docencia y de investigación con modalidades estrechamente vinculadas con el desarrollo social de sus de sus comunidades, tanto a nivel nacional como de las de sus respectivas regiones. La extensión universitaria -función específica de la universidad latinoamericana- ha ido perdiendo, lamentablemente, la significación social que los reformistas de 1918 se plantearon originariamente, función que se hace necesario reivindicar y fortalecer, como forma de desarrollar un conocimiento con pertinencia social.

- Solidaridad con el pueblo y los trabajadores: este eje de la Reforma Universitaria de 1918 profundiza el sentido social ya expresado en los anteriores y muestra el fuerte sentido de solidaridad con la sociedad y su pueblo con que fue planteado el modelo reformista. Lamentablemente, quizás este eje no haya sido desarrollado hasta ahora fuertemente y la universidad latinoamericana continúe manteniendo una concepción de carácter elitista, con muy escasas excepciones.

- Superación de las fronteras de la universidad: este eje complementa los anteriores -función social, compromiso con el cambio, solidaridad con el pueblo y los trabajadores- porque esos otros ejes solo pueden desarrollarse en una universidad que supere sus fronteras y que se brinde a la sociedad en forma plena, trabajando y produciendo conocimiento conjuntamente con el pueblo, que debería considerarse destinatario y productor colectivo del mismo. Es decir, superar el modelo -todavía vigente en muchas instituciones de América Latina- de una universidad cerrada en "su torre de marfil". 


\section{LA UNIVERSIDAD QUE TENEMOS}

Si los ejes e ideas centrales de la Reforma Universitaria de 1918 -recién planteados sintéticamente- se hubiesen desarrollado plenamente en estos cien años, la Universidad en América Latina sería muy diferente a la que actualmente tenemos. Como ya se señaló, los efectos de las dictaduras militares, de las políticas neoliberales en las últimas décadas y la ausencia de estrategias nacionales y regionales y de conducciones universitarias que hubiesen complementado, actualizado y perfeccionado las concepciones sobre Universidad de 1918, han provocado que la universidad actual en Argentina y en América Latina no se corresponda con los principios y lineamientos más deseables planteados por los estudiantes cordobeses hace un siglo.

La Universidad que actualmente tenemos en América Latina -salvo limitadas excepciones institucionales- puede caracterizarse de la siguiente manera: ${ }^{2}$

- Fuerte diversificación, segmentación y heterogeneidad de los niveles de calidad de las instituciones universitarias en América Latina, ya que en la región y en la mayoría de sus países existen universidades -la minoría- de un alto nivel académico y muchas otras -la mayoría- de muy bajo nivel. Esto afecta negativamente a sus estudiantes y graduados, la mayoría de ellos en este tipo de instituciones, pertenecientes a sectores sociales de bajos ingresos.

- Gran crecimiento del número de universidades privadas, muchas de ellas de muy bajo nivel académico. Actualmente en América Latina los estudiantes que concurren a universidades privadas constituyen alrededor del $60 \%$ de la matrícula universitaria total: en Brasil alcanza a más del $80 \%$. Las excepciones la constituyen Cuba -que no tiene universidades privadas, Uruguay, con alrededor del 10\% y Argentina, con aproximadamente el $15 \%$.

- Crecimiento inorgánico del número de universidades y de estudiantes, la mayoría de los cuales cursa carreras no vinculadas con las necesidades actuales y futuras de los respectivos países. En la Argentina la matrícula se concentra en las carreras tradicionales de las Ciencias Sociales y de las profesiones como abogacía, administración, contaduría,

2 Para una profundización de este análisis pueden consultarse - entre otros textos del autor- Norberto Fernández Lamarra $(2003,2010)$. 
etc. Existe un muy bajo número de estudiantes en carreras de Ciencias Básicas y de Tecnología.

- Escasa articulación entre la educación superior -universitaria y no universitaria- con el resto del sistema educativo, particularmente la enseñanza media.

- Falta de flexibilidad, innovación y actualización científica de los diseños curriculares y de los programas de las asignaturas.

- Escasa articulación entre las instituciones de educación superior con la sociedad, el trabajo y la producción y poca participación social en el desarrollo universitario.

- Rigidez -y obsolescencia en muchos casos - en los modelos organizativos y en las estructuras de gestión académica y afectando la necesaria flexibilidad para la planificación y el desarrollo universitario (FERNÁNDEZ LAMARRA, 2012).

- Políticas nacionales e institucionales de desarrollo con lineamientos excesivamente genéricos, sin atender adecuadamente las perspectivas de mediano y largo plazo y sin proyectos específicos para hacerlos efectivos.

- Escaso gasto público en investigación para el desarrollo $(\mathrm{I}+\mathrm{D})$, con la excepción de Brasil que destina algo más del 1\%. A nivel mundial, los países con mayor desarrollo destinan entre el 2\% y el 3\% de sus PBIs.

- Limitada democratización de la educación superior en América Latina: habiéndose registrado en las últimas décadas una expansión de la matrícula y del número de instituciones sin haberse modificado las condiciones estructurales de la desigualdad social, con dificultades de acceso y permanencia y con la persistencia de modelos universitarios diseñados para los sectores medios y altos y con fuertes desigualdades de formación en el nivel medio. Como lo afirma Ana María Ezcurra (2011), la masificación de la educación superior en América Latina registra brechas agudas en las tasas de graduación, según nivel socio-económico; es decir, esta socialmente condicionada, y agrega Ezcurra (2011), que predomina una perspectiva encubridora, que preserva el status quo, lo que implica una lógica de reproducción de la desigualdad cultural socialmente condicionada. La exclusión se registra dentro del sistema-se- 
gún José Dias Sobrinho (2008)- por la oferta de enseñanza precaria y de baja calidad en un alto número de instituciones de educación superior. Las instituciones de educación superior en algunos países de América Latina reciben nombres como el de "universidades garaje" en Colombia y "universidades patito" en México. En la Argentina -que tiene un sistema prácticamente de "libre ingreso" a las universidades públicasentre el $50 \%$ y el $60 \%$ de los ingresantes deserta habiendo cursado sólo el primer año o parte limitada del segundo, luego de haber permanecido varios años en las instituciones universitarias. Prevalece la concepción de que fallan los estudiantes, no las instituciones ni el sistema.

\section{UNIVERSIDAD, SOCIEDAD, GOBERNABILIDAD Y DEMOCRATIZACIÓN DE LA EDUCACIÓN SUPERIOR}

Los contextos socio-políticos predominantes en América Latina en los últimos años muestran un difícil proceso hacia el mejoramiento de las condiciones para asegurar la gobernabilidad democrática de cada uno de los países. Muchos de ellos están transitando complejas situaciones de transición y de consolidación gradual de la democracia, conjuntamente con los impactos de la globalización, de la imposición de las economías de mercado, de la internacionalización de las decisiones económicas, de las reformas del Estado de orientación neoliberal, de las crisis de las economías nacionales y de procesos crecientes de pobreza, exclusión social y marginalidad.

Conjuntamente con esto, los países de América Latina realizan esfuerzos, a veces en condiciones muy difíciles, para integrarse en bloques regionales -como, por ejemplo, MERCOSUR, Comunidad Andina de Naciones, Centroamérica y el NAFTA- y simultáneamente hacer frente a los desafíos de la nueva sociedad del conocimiento y de la tecnología.

La pertinencia político-institucional de los sistemas universitarios se define en términos de sus contribuciones al afianzamiento de la gobernabilidad democrática de la región en su conjunto y en cada uno de sus países. De ahí que la universidad debe trabajar efectivamente para alcanzar consensos básicos en materia de políticas públicas a través de procesos de concertación, para promover nuevas modalidades de representación social, para establecer canales institucionales para las demandas de participación social, para el desarrollo y aceptación colectiva de valores ético-morales en el marco de una cultura cívica democrática, para el desarrollo en sus estudiantes y graduados de actitudes y juicios críticos sobre instituciones, procesos y actores y para que la 
educación y la universidad se constituyan nuevamente en medios eficientes de movilidad e integración social.

La gobernabilidad democrática requiere de la mejor gobernabilidad de los sistemas educativos y de la universidad en términos de legitimidad, eficiencia y participación (PUELLES BENITEZ; URZÚA, 1996). La legitimidad plantea la necesidad de políticas educativas y universitarias establecidas a través de procesos de concertación, con planes y programas de carácter estratégico, teniendo en cuenta escenarios de largo plazo, con criterios en cuanto a la pertinencia de contenidos y calidad y con prioridades y metas en términos de equidad social y del financiamiento disponible.

La gobernabilidad democrática de los sistemas educativos y de las instituciones universitarias requiere asumir a la educación como una tarea de todos, con la centralidad del Estado, con una actitud pluralista y negociadora y con decisiones adoptadas en forma participativa y consensuada en el marco de una nueva relación con el Estado y la sociedad en que se transite del Estado Docente a la Sociedad Educativa y del Conocimiento.

La eficiencia exige que la educación superior -así como el conjunto del sistema educativo- brinden respuestas satisfactorias a las necesidades de la población escolar y universitaria y a la sociedad en su conjunto. Esto requiere buena gestión de los procesos y de los recursos humanos, financieros y materiales involucrados, la mayor profesionalización de docentes y no docentes y un proceso permanente de evaluación de resultados y de impacto.

La participación, como requisito de la gobernabilidad democrática, plantea la necesidad de promover un mayor protagonismo de la sociedad organizada en la fijación de las políticas de educación superior y en la conducción de las instituciones universitarias. Así como la Reforma Universitaria de 1918 estableció -como ya se ha señalado- la participación imprescindible de los claustros de docentes y estudiantes y luego de los graduados en la conducción de las universidades, se deben replantear estos criterios de manera de integrar la participación de representantes de las organizaciones sociales y del Estado, siempre en el marco de una autonomía universitaria fortalecida. Esto exige democratización de los procesos participativos intra y extra universitarios y la promoción de nuevas modalidades de participación y de representación.

Los debates en cuanto al fortalecimiento de la gobernabilidad democrática en las instituciones universitarias y su contribución a la necesaria gobernabilidad democrática de los países de la región están prácticamente ausentes del ámbito de nuestras universidades. Estos debates son imprescindibles para fortalecer la pertinencia política e institucional de la educación superior en 
la región y evitar conflictos, en el seno de las universidades, que las afecten seriamente.

Las exigencias de la gobernabilidad democrática y de la sociedad del conocimiento y de la tecnología, requieren plantearse cambios muy significativos en las propias universidades y en la relación entre éstas y la sociedad.

La universidad debería constituirse en el ámbito principal de creatividad e innovación para sí misma y para la sociedad en su conjunto. Para ello debe superar los problemas de su escasa articulación con la sociedad, el trabajo y la producción; de su aislamiento con el resto del sistema educativo; de sus modelos académicos y de gestión tradicionales, con una muy limitada profesionalidad; de la rigidez de sus estructuras académicas; de la escasez de estudios, investigaciones y reflexión sobre sí misma y sobre la educación superior en su conjunto.

De ahí, que sea necesario plantearse nuevas modalidades para la docencia, con nuevos objetivos para la formación, con diseños curriculares innovadores que atiendan los requerimientos académicos y los de la sociedad con una perspectiva de futuro, con renovadas metodologías de enseñanza-aprendizaje y con un enfoque de educación permanente.

Para esto, los planteamientos de Miguel Ángel Escotet (1997) para el cambio universitario constituyen una base útil para el debate. Desarrolla tres ejes: universidad para la reflexión en la acción (reflexión anticipatoria; formación centrada en el sujeto que aprende y orientada a "aprender a emprender", a "aprender a cuidar" y a "seguir aprendiendo", cooperación interuniversitaria como entramado de la "multiuniversidad; etc.); universidad para la diversificación (diversificación de los que aprenden; de la interdisciplinariedad, áreas y disciplinas; de los procesos de enseñanza-aprendizaje; de las instituciones; de las acreditaciones, disciplinas y títulos; de la formación, etc.); y universidad para la flexibilidad (flexibilidad en las estructuras del currículo, en la transferencia de conocimientos, en los sistemas de acreditación, en las formas de financiamiento, en los mecanismos de reforma y cambio, etc.). Es decir, afirma Escotet (2002), una universidad para la innovación, orientada hacia el logro permanente de una vigorosa capacidad de creación y cambio y una universidad para el hombre y su medio, orientada al progreso económico y social en el marco de un desarrollo sostenible al servicio de del hombre y su habitat. También plantea la necesidad de "una universidad que contribuya a ampliar la capacidad de su sociedad para convivir en un mundo interdependiente y cuyo futuro es patrimonio de la sociedad global". 
Las características de la sociedad del conocimiento y de la tecnología y los desafíos actuales que se le presentan a las instituciones de educación superior, deben llevar a replantearse el rol de la universidad en cuanto a la creación, gestión y difusión de los nuevos conocimientos, por lo que las funciones de investigación y de extensión y transferencia deben articularse fuertemente con la docencia, enriqueciéndose mutuamente. Sólo de esa manera, la universidad puede ir cumpliendo los roles que la nueva relación educación superior-sociedad le exige.

Ya se ha planteado la necesidad de que las políticas de educación superior y las instituciones universitarias contribuyan efectivamente al mejoramiento de la gobernabilidad y, en general, a una mayor democratización de la sociedad, favoreciendo estrategias que tiendan a superar $-\mathrm{o}$, por lo menos, a disminuir- las iniquidades sociales y los procesos de crecimiento de las desigualdades en términos de pobreza y marginalidad.

La importancia y significación de la sociedad del conocimiento y de la tecnología requieren de estrategias específicas para evitar de aquí al futuro la aparición y el crecimiento de una nueva modalidad de marginalidad: la de los "marginales de la sociedad digital". Actualmente los que acceden a Internet son todavía una minoría concentrada en los países desarrollados: alrededor de mil millones de personas. De esos se estima que en América Latina el número de personas que actualmente accede a Internet es del orden de 40 millones, o sea sólo el 4\% con respecto a los países desarrollados. De esos 40 millones, la casi totalidad corresponde a los sectores medios y altos de estudiantes de educación superior, profesionales, empresarios y técnicos superiores (BRUNNER, 2000).

La evolución de la educación superior en la última década ha intensificado su carácter elitista. El acceso a las instituciones de educación superior, públicas y privadas de mejor calidad ha quedado reservado casi exclusivamente a los jóvenes de clase media y media alta, provenientes de escuelas medias públicas y privadas de buena calidad. Los de menos nivel social, provenientes de escuelas medias públicas de más baja calidad, han tenido que asistir a instituciones de educación superior, universitarias y no universitarias de muy baja calidad, en general privadas, cuyo número, lamentablemente, ha crecido sustantivamente.

Se está asistiendo a la generación de un nuevo proceso de "fraude", porque si los estudiantes de menor nivel social egresan ese tipo de instituciones de menor nivel de calidad -luego de un gran esfuerzo por parte de sus familias y de ellos mismos- sus títulos tendrán una más baja valoración en 
el mercado de trabajo; es decir, menos posibilidades ocupacionales y menores salarios. Este grave problema de desigualdad social agravada por los sistemas educativos y universitarios debe constituirse en centro de debate, de nuevas políticas y de profundas reformas e innovaciones en la educación superior latinoamericana, como ya ha sido planteado en el punto anterior de este artículo. Esto no sólo es necesario por razones éticas y políticas sino, también, por la alta correlación entre la educación superior y la transformación productiva de las sociedades latinoamericanas. Según la UNESCO (2003), la producción y acumulación de conocimientos son la fuerza principal del desarrollo en el actual contexto de la globalización (FERNÁNDEZ LAMARRA, 2009).

\section{LA INNOVACIÓN COMO POLÍTICA Y ESTRATEGIA CENTRAL EN LA UNIVERSIDAD}

Teniendo en cuenta -como ya lo he expresado- la importancia y significación de las estrategias innovadoras para las políticas y el desarrollo de universitario vinculados al conocimiento, el Núcleo Interdisciplinario de Formación y Estudios para el Desarrollo de la Educación (NIFEDE) de la Universidad Nacional de Tres de Febrero -que dirijo- viene desarrollando en los últimos dos años una línea de investigación en torno a la innovación en la educación superior (Proyecto PICTO/UNTREF-4/73). La investigación se planteó como estudio de casos y toma como universo las experiencias de innovación de universidades públicas argentinas. El trabajo de investigación se organizó a partir de cuatro fases diferenciadas: la construcción de una muestra teórica de universidades, la identificación de innovaciones significativas desarrolladas en ellas, la caracterización histórico situacional de una selección de tales innovaciones y el estudio de los grupos involucrados en su emergencia y desarrollo. Para posibilitar esta identificación, inicialmente se llevaron a cabo entrevistas en profundidad a más de veinte colegas con diferentes enfoques académicos y distintos niveles de vinculación con la universidad pública argentina.

\subsection{APROXIMACIONES PARA UNA DELIMITACIÓN CONCEPTUAL DE LA INNOVACIÓN}

La indagación bibliográfica permitió comprender que el término innovación ha sido utilizado de diversos modos y con cierta ambigüedad, e intercambiado con otros afines (VILLA SÁNCHES; ESCOTET; GOÑI ZABALA, 
2009). Particularmente en el ámbito educativo, un primer problema detectado en la indagación teórica se vincula con el concepto mismo y con la escasez de un marco teórico suficientemente desarrollado y compartido que permita identificar qué es, o no, innovador, y que proporcione un marco de referencia para el desarrollo de innovaciones en el país y en la región. Otra cuestión que surge es la diversidad de sentidos que se le otorga al concepto; diversidad advertida también a partir de los testimonios registrados en las entrevistas durante el trabajo de campo inicial de la investigación.

Un concepto que se encuentra estrechamente vinculado al de innovación es el de transformación. Las estrategias para la transformación de la educación superior en América Latina han sido variadas y diferentes. Por ello se hace necesario comenzar su análisis tratando de encontrar categorías que permitan clasificarlas. En un estudio que realizamos hace alrededor de 30 años atrás (FERNÁNDEZ LAMARRA; AGUERRONDO, 1983) planteamos que una clasificación posible sería diferenciarlas de acuerdo a su ámbito de aplicación. Hay transformaciones que tratan de abarcar la totalidad del sistema educativo y/o universitario, otras que intentan transformar determinada parte de él, y otras que se circunscriben a un ámbito institucional específico o a varias instituciones. Otra categoría identificada al respecto por estos autores es el contenido de la propuesta; el cruce de estas dos categorías (ámbito y contenido) permitiría identificar propuestas que abarcan la transformación de todo el sistema, denominadas habitualmente "reformas" y que pueden ser "de la educación" o estructurales cuando intentan transformar la estructura del sistema educativo tradicional o bien, "educativas" o generales cuando sus contenidos apuntan básicamente a perfeccionarlo.

Para avanzar en el estudio de la noción de innovación resulta pertinente también distinguir entre cambio e innovación. Innovación no es sinónimo de toda modificación -o cambio- en alguno de los componentes de un sistema. Supone un «cambio» orientado y fundamentado, dentro de un proyecto. Una innovación es una mejora que se puede medir, que es resultado de una elección y un desarrollo deliberados, que es duradera y no es probable que ocurra frecuentemente (MORRISH, 1976).

Desde otras múltiples perspectivas también se ha estudiado el concepto de innovación. Las primeras investigaciones surgieron en el ámbito de lo empresarial con estudios como el realizado por Druker (1985). Ahora bien, en el campo de lo educativo, a lo largo de las últimas décadas se han desarrollado varias definiciones y clasificaciones de los tipos de innovación en la educación superior. Hasta finales de la década de los ochenta la investigación en el tema 
estuvo fundamentalmente centrada en el análisis a nivel micro de metodologías y procesos innovadores. Se consideraba que la innovación y el cambio se producían de forma personal a través de las modificaciones realizadas por determinados profesores. La creciente institucionalización de los esfuerzos por la innovación durante los últimos años ha dado paso a lo que Hannan y Silver (2005) denominan "innovación guiada". Esta no niega el proceso personal pero centra la atención en la innovación dirigida e incentivada desde las instituciones. Otro estudio sobre la cuestión de la innovación en la educación superior ha sido realizado por Cuban (1999) quien considera que hay que tener presente dos criterios: la penetración y la efectividad. En el primer caso, hay que evaluar si el cambio ha modificado realmente las formas de enseñanza-aprendizaje. La efectividad se refiere al impacto real en la mejora del aprendizaje. En un estudio realizado sobre la enseñanza universitaria, Zabalza (2003) define innovar como no solamente hacer cosas distintas sino hacer cosas mejores.

\subsection{LAS EXPERIENCIAS DE INNOVACIÓN EN LA UNIVERSIDAD PÚBLICA ARGENTINA INCLUIDAS EN LA INVESTIGACIÓN}

A fin de relevar experiencias de innovación de carácter "endógeno" en las universidades públicas argentinas en diversos niveles de la organización (institucional, de unidad académica o de grupos de trabajo) y en las distintas dimensiones de su gestión (institucional, académica o administrativa) así como para registrar las condiciones de producción y las dinámicas de desarrollo de esas experiencias innovadoras y el lugar de los actores universitarios en su construcción, el NIFEDE/UNTREF desarrolló -como ya se señaló- entre 2012 y 2014 el estudio "Innovación en las universidades nacionales. Aportes para la comprensión de aspectos endógenos que inciden en su surgimiento y desarrollo"'.

El carácter endógeno de las innovaciones se refiere a las experiencias que la Universidad ha desarrollado, a lo largo de su historia e -incluso- en condiciones de profunda crisis, no como respuesta a la incidencia de políticas públicas específicas sino avanzando más allá de los límites marcados por ideologías hegemónicas o por sus propios paradigmas. Muchas veces, incluso, contrariando las políticas en desarrollo. En ocasiones estas innovaciones se han relacionado con la necesidad o el interés de diversos grupos que las

3 La investigación fue desarrollada en el marco del Programa PICTO UNTREF financiado por el Ministerio de Ciencia, Tecnología e Innovación Productiva de la Nación y la UNTREF. 
impulsan, orientados por su adhesión a proyectos y valores institucionales, académicos o disciplinares que los comprometían ética, política y/o ideológicamente.

A partir de estas proposiciones, la investigación profundizó el relevamiento y el estudio de una serie de casos correspondientes a universidades públicas representativas de distintos momentos históricos en la constitución del sistema universitario argentino y -como se ha señalado- en diferentes ámbitos de la organización y de la gestión que han logrado incidir favorablemente en desarrollos de las capacidades institucionales de formación, investigación y extensión, alcanzando reputación institucional, nacional e internacional.

En este sentido, en su desarrollo, la investigación identificó cinco dimensiones relevantes sobre las cuales profundizó, posteriormente, el estudio de las innovaciones:

a) El acceso a los estudios;

b) Las innovaciones pedagógicas -en relación con el curriculum y la enseñanza-;

c) La relación de la universidad con el medio social;

d) La institucionalización de la investigación y el desarrollo; y

e) El desarrollo de nuevas estructuras de organización y gobierno.

En la primera dimensión el foco se puso en las estrategias institucionales centradas en una mayor democratización del sistema a partir del proceso de masificación de la educación superior registrado en la Argentina, en especial a partir de la creación de nuevas universidades nacionales, en especial las creadas en las últimas dos décadas en el denominado Gran Buenos Aires. La investigación analizó las experiencias llevadas a cabo por parte de dichas universidades, que componen la Red de Universidades del Conurbano Bonaerense (RUNCOB) implementada a partir de 2007 tendiente a promover una mejor adaptación de los estudiantes a los estudios universitarios, lo que redundaría en una democratización no sólo en el acceso a los estudios, sino también en la permanencia y egreso ${ }^{4}$.

El interés del caso se basa en el hecho de tratarse de un conjunto de nuevas universidades creadas con el fin de atender el constante crecimiento pobla-

4 Esta concepción amplia de la democratización de la educación superior aún representa un problema central a ser resuelto en el país así como en la mayoría de los países de América Latina, especialmente afectando grupos sociales desfavorecidos (FERNÁNDEZ LAMARRA; COSTA DE PAULA, 2011; RAMA, 2006), tanto por su condición socio-económica como por otros factores asociados, tales como los geográficos, étnico-raciales, de género y/o físicos. 
cional del Conurbano de la Provincia de Buenos Aires, que presenta tanto los mayores índices de crecimiento poblacional como de desigualdad social. Además atienden un perfil de estudiante novedoso para el resto del sistema educativo: principalmente, son la primera generación familiar que se incorpora a la universidad, el estudio no es su principal actividad y no son jóvenes recién egresados de la escuela secundaria. Tienen en común también una nueva perspectiva institucional de caracterización y abordaje de sus estudiantes, frente a los cuales plantean sistemas no selectivos de ingreso y de fuerte acompañamiento, cargando en la responsabilidad institucional la efectiva inclusión de sus aspirantes y alumnos.

Asimismo, se relevó la experiencia que desde 1985 ha desarrollado la Universidad de Buenos Aires al implementar el Ciclo Básico Común (CBC) aún vigente y que, aunque no ha sido replicado como tal por otras instituciones, es el sistema de mayor alcance considerando su envergadura y su sostenimiento institucional por casi 30 años.

Respecto de las innovaciones pedagógicas vinculadas al curriculum y a la enseñanza, el Proyecto indagó aquéllas tendientes a incorporar espacios y prácticas docentes multidisciplinares o de aprendizajes prácticos que promocionaran conocimientos transversales y relacionados con el "afuera" de la universidad, o bien sobre la formación docente de los académicos para que cumplan con estos objetivos. Con la cooperación de la Prof. Clotilde Yapur de la Universidad Nacional de Tucumán, la investigación analizó el cambio en el modelo de formación de médicos llevado a cabo por esa Universidad a partir de 1988, modificando el eje de su preparación profesional hacia la prevención y la promoción comunitaria sobre un modelo multidisciplinario de atención. Esta experiencia impactó posteriormente en el resto del sistema universitario argentino.

En cuanto a la relación de la universidad con el medio social, la universidad argentina se caracterizó por su perfil "endógeno" y ya una de las demandas de la Reforma Universitaria -como ya sido señalado- fue la conexión del mundo académico con la sociedad y sus requerimientos. La extensión, como función irrenunciable de las universidades durante mucho tiempo, se extendió como una función subsidiaria de las demás. Sin embargo, a partir de ciertos actores fundamentales en la segunda mitad del siglo XX, se comenzaron a detectar actividades de trabajo académico en lugares y con agentes sociales no tradicionales, muchos de éstos, de sectores populares. Estas acciones han constituido a la extensión y al contacto con el medio como un ambiente dinámico en la producción y transmisión del conocimiento, cumpliendo directamente con funciones sociales pero también transformando las formas y mode- 
los de plantear la investigación y la docencia. También los proyectos de I+D, orientados a la atención de necesidades o problemas sociales, productivos, ha formado parte de estrategias de la universidad en su relación con el entorno.

El estudio desarrollado para analizar esta temática -de gran significación para la relación Universidad-Sociedad-Conocimiento- profundizó el caso del Centro para la Transferencia de los Resultados de la Investigación (CETRI) creado por la Universidad Nacional del Litoral (UNL) en 1993 con el objetivo de transferir al medio los resultados de la investigación, la generación de las actitudes emprendedoras en los estudiantes, la generación de empresas de base tecnológica y la generación de nuevos emprendimientos a partir de la comunidad universitaria. En otras palabras, el CETRI forma parte de la política de la UNL de generación de conocimientos vinculados con el medio socioproductivo de la región a fin brindar un apoyo sustentable a la región en que se inscribe. Su grado de desarrollo es considerado como una referencia nacional por diversos actores del sistema.

La dimensión de institucionalización de la investigación y el desarrollo se estudió a través del Programa de Promoción de la Ciencia y la Tecnología de la Universidad de Buenos Aires, más conocido por su acrónimo UBACYT. Aunque a nivel internacional el tradicional modelo continental de universidades tuvo un límite en la promoción de la investigación con el inicio de la masificación del sistema -y el caso argentino no estuvo exento de esto-, se han podido detectar innovaciones en relación a como promover la función de investigación y desarrollo, otorgándole una necesaria nueva dinámica de dicha función de producción del conocimiento. El Programa UBACyT, creado en 1987, es una de las iniciativas institucionales identificadas como innovadoras y que ha tenido un fuerte impacto en el resto de las universidades nacionales del país, incluso en el ámbito del Ministerio de Educación de la Nación 5 .

Finalmente, la dimensión que consideró el desarrollo de nuevas estructuras de organización y gobierno, estudió el surgimiento de experiencias innovadoras que rompieran el tradicional modelo organizativo de las universidades públicas argentinas basado en el esquema Facultades-carreras-cátedras. Esta característica tradicional tuvo problemas para generar espacios innovadores y

5 En el año 1994 surgió el Programa de Incentivos a los Docentes Investigadores del Ministerio de Educación, orientado a los profesores de universidades nacionales. El Programa de Incentivos implica un plus salarial a los docentes que participaban proyectos evaluados externamente (es decir, aquéllos con el formato UBACYT). Esto significó la duplicación de los proyectos existentes. Se incorporaron áreas que antes estaban ausenten en el UBACYT porque no necesitaban financiamiento para realizar un proyecto (por ejemplo Matemática, de carácter teórico) ya que al existir un plus salarial se requirió su inclusión en el Programa UBACYT. El crecimiento del programa verificó un incrementó del 89\% en la cantidad de solicitudes y de un $64 \%$ de los proyectos financiados. 
responder a nuevas realidades científicas, sociales y a las necesidades geográficas de nuevas instituciones.

El Proyecto estudió -también- un nuevo modelo organizativo surgido a partir de las universidades creadas en el marco del "Plan Taquini" a finales de la década de 1960 con el objetivo de reestructurar la educación superior en Argentina descomprimiendo a las grandes universidades nacionales -ubicadas en la ciudad de Buenos Aires (la Universidad de Buenos Aires) y en las capitales de las provincias más importantes-. De esta manera se fue cubriendo gradualmente las demandas de educación superior en el interior del país, abarcando a todas las provincias y en algunas de ellas al interior de su territorio. Asimismo, con estas nuevas universidades se procuraba promover la investigación científica y tecnológica asociada a problemáticas locales, descentralizando su desarrollo. En la investigación, específicamente, se abordó el caso de la Universidad Nacional de Río Cuarto, en la provincia de Córdoba, creada en 1971.

\subsection{RESULTADOS Y CONCLUSIONES DE LA INVESTIGACIÓN}

Actualmente el equipo de investigación del NIFEDE está concluyendo sus trabajos y elaborando sus principales conclusiones. Se espera que las mismas -que podrán profundizarse con otros estudios posteriores por parte del NIFEDE y de investigadores de otros centros académicos- contribuyan a esclarecer las contribuciones de las propias universidades pública argentinas para llevar adelante políticas institucionales que promuevan la innovación en todos los aspectos como estrategia permanente y no sólo como excepción. Se hace necesario en el sistema educativo y, muy especialmente en el universitario, convertir a las políticas innovadoras como práctica permanente, promoviéndolas desde las políticas nacionales y desde las estrategias institucionales, ya que instituciones educativas y universitarias de carácter innovador y emprendedor son la base para formación de graduados con espíritu innovador y emprendedor, que son la base para la implementación de políticas que promuevan al conocimiento científico y tecnológico con carácter prioritario para el desarrollo de nuestros sociedades nacionales (MORA; VIEIRA, 2009).

\section{PRINCIPALES DESAFÍOS PARA A LAS POLÍTICAS Y LAS INSTITUCIONES DE EDUCACIÓN SUPERIOR}

Durante el desarrollo de este artículo se han ido planteando algunos de los principales desafíos que las universidades deben asumir-desde mi perspecti- 
va- frente a las nuevas responsabilidades que se les plantean en lo político, en lo social y en lo académico en América Latina, frente a las exigencias cada vez más urgentes de la Sociedad del Conocimiento y de las Nuevas Tecnologías. Estos desafíos -y otros muchos más que surjan de los colegas, de las propias instituciones universitarias y de la sociedad a través de sus organizaciones representativas- deben ser tomados en consideración para los necesarios debates que deberán sostenerse para asegurar que nuestras instituciones educativas y universitarias respondan a los requerimientos políticos, sociales y académicos, tanto de largo plazo como más inmediatos. Es de esperar que estos debates posibiliten estructurar entre todos un nuevo proyecto de Reforma Universitaria que-como homenaje a los estudiantes de Córdoba de 1918- refleje los lineamientos para atender dichos requerimientos. Ojalá este nueva propuesta de Reforma Universitaria para el siglo XXI pueda elaborarse antes de alcanzar -ya muy pronto- el centenario de la Reforma Universitaria.

En este último punto se intentará resumir algunas de las propuestas y desafíos que he ido planteando a lo largo de este artículo.

Desde la perspectiva de las políticas para la educación superior pueden plantearse, entre otros, los siguientes desafíos (FERNÁNDEZ LAMARRA, 2010):

- Diseñar y ejecutar estas políticas en un marco amplio y participativo de consenso entre todos los actores pertinentes intra y extra universitarios, que posibilite el involucramiento de todos los actores universitarios, de la sociedad y de los ámbitos gubernamentales en estas definiciones que son decisivas para el futuro de la universidad, de la sociedad y de cada uno de los países

- Contribuir efectivamente a mejorar la gobernabilidad democrática y la calidad de la democracia vigente en nuestros países y, a su vez, perfeccionar la gobernabilidad de cada una de las instituciones universitarias, ya que este un compromiso ineludible de las universidades en relación con sus respectivas sociedades nacionales.

- Articularse más fuertemente con la sociedad y sus organizaciones representativas tendiendo a establecer una nueva alianza sociedad-universidad, que permita asumir sus demandas y posibilitar su participación efectiva en el diseño y desarrollo de las políticas sectoriales e institucionales, generando nuevas modalidades de gobierno y de gestión de las instituciones universitarias. 
- Reafirmar las concepciones de la autonomía universitaria y del cogobierno y replantearlas en el marco de los nuevos desafíos de la sociedad y de su gobernabilidad, evitando que la autonomía conduzca al aislamiento de las instituciones universitarias en relación con sus sociedades nacionales y regionales y buscando que las mismas se articulen fuertemente con las demandas sociales actuales y futuras.

- Articularse con las políticas y planes del conjunto de la educación y del sistema científico-tecnológico, de manera de responder en forma pertinente a sus desafíos, asumiendo efectivamente que la educación superior integra los sistemas educativos y científicos nacionales y debe trabajar conjuntamente con ellos.

- Definir las políticas sectoriales en un marco de mediano y largo plazo, de manera de atender los requerimientos tanto los inmediatos como los mediatos, ya que esto se constituye cada vez más en un imperativo ineludible.

- Articularse con los sectores del trabajo y la producción de manera de contribuir a su mejoramiento tecnológico y a su funcionamiento en el marco de los requerimientos globales, regionales y nacionales, organizando para ello programas y proyectos de formación permanente, de investigación y de transferencia y proyección a la sociedad, según sus necesidades actuales y futuras.

- Asumir una concepción estratégica de reforma y cambio, en un marco de planeamiento y gestión de carácter innovador, en lo institucional, en lo académico, en lo organizativo y en lo pedagógico.

- Contribuir a atender prioritariamente los requerimientos de los sectores sociales pobres, de manera de posibilitarles su mejor inserción en el sistema educativo y en la educación superior y contribuyendo efectivamente a la democratización tanto de la sociedad como de las propias instituciones educativas.

- Tender a superar la fragmentación actual de la educación superior y sus niveles de gran heterogeneidad en cuanto a calidad y pertinencia, promoviendo procesos de articulación y convergencia tanto a nivel nacional como en cada una de sus regiones, a través de redes interinstitucionales de trabajo conjunto .

- Asumir que el conocimiento es un factor decisivo para el desarrollo equilibrado de los países y que las universidades deben contribuir a 
incrementarlo fuertemente con sus acciones de formación, investigación y transferencia y deben utilizarlo efectivamente para impulsar su propio auto desarrollo y el de la sociedad en su conjunto.

- Incrementar sustantivamente los recursos financieros para la educación superior y para la investigación científico-tecnológica y mejorar los procesos de asignación y utilización de los disponibles en función de prioridades y requerimientos nacionales, regionales e institucionales.

- Aprovechar plenamente las posibilidades que brindan la NITCs, de manera que estén al servicio de una política democratizadora de educación superior para todos y que posibiliten articular efectivamente las acciones de educación presencial y virtual, aprovechando efectivamente las ventajas de cada modalidad y superando sus propias limitaciones.

- Establecer regulaciones y normativas -de común acuerdo con otros países de la región- que tiendan a controlar en función de las necesidades nacionales las propuestas y ofertas de educación superior de carácter trasnacional, que pueden afectar fuertemente el desarrollo de los sistemas universitarios nacionales.

- Favorecer los procesos de integración regional y subregional de manera que los sistemas universitarios se incorporen a una alianza estratégica tendiente a construir una Comunidad Latinoamericana de Naciones y un Espacio Común Latinoamericano de Educación Superior, con una estrategia que otorgue prioridad a la cooperación académica y el desarrollo del conocimiento Sur-Sur y que a partir de ese eje se articule la relación Norte-Sur. Para ello será necesario promover y organizar procesos de intercambio de estudiantes de grado y de posgrado, de profesores e investigadores y organizar programas académicos de formación de grado y de posgrado y de investigación y transferencia a través de redes interuniversitarias integradas por instituciones universitarias y centros de investigación de América Latina.

En cuanto a las instituciones de educación superior deberían trabajar intensamente en el marco de estas estrategias para el sistema en su conjunto y desarrollar políticas, planes y proyectos institucionales que tiendan a concretarlas. Entre los desafíos más significativos para las instituciones universitarias pueden plantearse -entre muchos otros- los siguientes: 
- Los procesos de evaluación institucional y de acreditación deben tener un carácter permanente y participativo y constituirse en la base para los cambios e innovaciones institucionales de mejoramiento de la calidad y de su pertinencia académica y social.

- Estos procesos deben facilitar el tránsito de la "cultura de la evaluación" a la de una "cultura de gestión responsable, autónoma, pertinente y eficiente" a nivel de cada institución universitaria, evitando que éstos sean procesos aislados y de carácter meramente formal

- Debe tenderse a contar con estructuras institucionales innovadoras, con nuevas modalidades de conducción y de toma de decisiones, de carácter democrático y con amplia y decisiva participación de todos los actores intra y extra universitarios.

- El desarrollo institucional, a mediano y largo plazo, debe estar basado en programas y proyectos específicos de carácter estratégico, articulados con los requerimientos nacionales y de cada región, establecidos con una orientación prospectiva.

- Las estructuras organizativas deben ser flexibles e innovadoras y basarse en una reingeniería en profundidad de los actuales procesos de toma de decisiones, planificación, gestión, seguimiento y evaluación, de manera de constituirse en base para el estudio, debate y adopción de los imprescindibles cambios requeridos

- El funcionamiento de las instituciones universitarias debe articularse -desde su autonomía- con los sectores del trabajo y la producción, con las organizaciones sociales y con todos los ámbitos públicos y privados pertinentes.

- Debe asumirse una concepción institucional de calidad que se base en una "construcción social", de carácter gradual y colectivo, pertinente, basada en un proceso de reflexión y debate intra y extra universitario.

- Las instituciones universitarias deben funcionar en un ámbito de creatividad, de innovación, de mirada hacia el futuro. Para ello deben planificar estratégicamente, ejecutar con responsabilidad, hacer seguimiento y control y evaluar como proceso permanente y autorregulado.

- Flexibilizar y profesionalizar las estructuras de conducción y gestión institucional, académica y administrativa con sentido integrador y utilizando las tecnologías más eficientes. 
- Articular e interrelacionar efectivamente las funciones de docencia, investigación y extensión y transferencia, en el marco de las prioridades institucionales.

- Desarrollar acciones articuladas de formación con carácter presencial y a distancia, de manera de aprovechar desde lo pedagógico, las ventajas y fortalezas de cada uno de ellos y superar sus limitaciones.

- Asociarse y establecer redes académicas con otras instituciones del país, de la región y de otras regiones de manera de estimular el intercambio de estudiantes, docentes e investigadores y de acordar programas articulados de docencia, investigación y extensión.

Estos lineamientos y desafíos, tanto en el ámbito de las políticas como en el del desarrollo institucional, se plantean -como ya ha sido señalado- para contribuir a construir una nueva agenda que facilite y promueva un debate amplio en la región, en cada país y en cada universidad, sobre los retos y expectativas que se le presentan a la educación superior en su conjunto y a cada una de sus instituciones. La pertenencia, eficiencia y el carácter creativo con que se encaren estas nuevas responsabilidades políticas, sociales y académicas de las universidades favorecerán o no sus mejores y deseables contribuciones a un pleno desarrollo en democracia y con justicia social de los países de América Latina y a responder de manera innovadora a la exigencias de la Sociedad del Conocimiento. Asimismo, posibilitarán la construcción colectiva de una nueva Reforma Universitaria para América Latina, que rinda homenaje a la Reforma de 1918, la actualice, la profundice y que haga posible que la Universidad Latinoamericana responda efectivamente a los requerimientos actuales y futuros de la región en lo político, en lo social y en lo académico.

\section{REFERENCIAS}

BRUNNER, J. J. Educación: escenarios del futuro. Nuevas tecnologías y sociedad de la información. Santiago de Chile: PREAL / Fundación Chile, 2000.

CUBAN, L. Change without reform in university currículo, teaching, and research. New York: Teachers College Press, 1999.

DIAS SOBRINHO, J. Calidad, pertinencia y responsabilidad social de la universidad latinoamericana y caribeña. En: GAZZOLA, A. L.; 
DIDRIKSSON, A. Tendencias de la educación superior en américa latina y el Caribe. Caracas: IESALC-UNESCO, 2008.

DRUCKER, P. Innovation and entrepreneurship. New York: Harper \& Row, 1985.

ESCOTET, M. A. Universidad y devenir. Buenos Aires: Lugar Editorial, 1997.

. Desafíos de la educación superior en el siglo de la incertidumbre. Cuaderno de Investigación en la Educación, San Juan, Puerto Rico, n. 18, 2002. Recuperado de: $<$ http://cie.uprrp.edu/cuaderno/ediciones/18/c18art1. htm>. Acceso en: 15 maio 2014.

EZCURRA, A. M. Igualdad en educación superior. Un desafío mundial. Los Polvorines: Universidad Nacional de General Sarmiento - IEC/ CONADU, 2011.

FERNÁNDEZ LAMARRA, N. La educación superior argentina en debate. Situación, problemas y perspectivas. Buenos Aires: IESALCEUDEBA, 2003.

FERNÁNDEZ LAMARRA, N. Universidad, sociedad y calidad en América Latina. En: FERNÁNDEZ LAMARRA, N. (Comp.). Universidad, sociedad e innovación. Una perspectiva internacional. Buenos Aires: EDUNTREF, 2009.

FERNÁNDEZ LAMARRA, N. Hacia una nueva agenda de la educación superior en América Latina. México-DF: ANUIES, 2010.

FERNÁNDEZ LAMARRA, N. Las políticas y la gestión universitaria en América Latina. Situación, problemas y perspectivas. En: FERNÁNDEZ LAMARRA, N. (Comp.). La gestión universitaria en América Latina. Coronel Oviedo, Paraguay: Universidad Nacional de Caaguazú, 2012. FERNÁNDEZ LAMARRA, N.; AGUERRONDO, I. Las reformas de la educación en America Latina; análisis de algunos procesos nacionales. Mimeo, 1983.

FERNÁNDEZ LAMARRA, N.; PEREZ CENTENO, C. . La autonomía universitaria en Argentina y América Latina. En: ROSARIO, V. M.; MARÚM ESPINOSA, E.; ALVARADO NANDO, M. (Coords.). La autonomía universitaria a debate. Una visión desde América Latina. Jalisco: Editorial Universitaria, Universidad de Guadalajara, 2010. 
FERNÁNDEZ LAMARRA, N.; COSTA DE PAULA, M. F. (Coord.). La democratización de la educación superior en américa latina. Límites y posibilidades. Buenos Aires: EDUNTREF, 2011.

FERNÁNDEZ LAMARRA, N.; COPPOLA, Natalia. La reforma del 18 y la autonomía universitaria. En: TEODORO, António; BELTRÁN, José (Comp.). Sumando voces. Ensayos sobre Educación Superior en términos de igualdad e inclusión social. Buenos Aires: Miño y Dávila Editores, 2014. HANNAN, A.; SILVER, H. La innovación en la enseñanza superior. Madrid: Narcea, 2005.

MORA, José-Ginés; VIEIRA, María José. Gobernanza universitaria y emprendedurismo: un estudio empírico. En: FERNÁNDEZ LAMARRA, N. Universidad, sociedad e innovación. Una perspectiva internacional. Buenos Aires: EDUNTREF, 2009.

MORRISH, I. Cambio e innovación en la enseñanza. Madrid: Anaya, 1976.

PUELLES BENÍTEZ, M.; URZÚA, R. Educación, gobernabilidad democrática y gobernabilidad de los sistemas educativos. Revista Iberoamericana de Educación, n. 12, 1996. Recuperado de: $<$ http://www. rieoei.org/oeivirt/rie12a05.htm>. Acceso en: 15 maio 2014.

RAMA, C. La tercera reforma de la educación superior en América Latina. Buenos Aires: Fondo de Cultura Económica, 2006.

UNESCO. Forum Occasional Paper Series Paper no. 4 / S Desafíos de la Universidad en la Sociedad del Conocimiento, Cinco Años Después de la Conferencia Mundial sobre Educación Superior, 2003.

VILLA SÁNCHEZ, A.; ESCOTET M. A.; GOÑI ZABALA, J. J. Elementos para un modelo de innovación de las instituciones de educación superior. En: FERNÁNDEZ LAMARRA, N. (Comp.). Universidad, sociedad e innovación. Una perspectiva internacional. Buenos Aires: EDUNTREF, 2009.

ZABALZA, M. A. Innovación en la enseñanza universitaria. Revista Contextos Educativos, Espanha, n. 6-7, p. 113-136, 2003. 
